References

[1] "Abnormal situation management - effective automation to improve operator performance", Honeywell Inc., Philadelphia, 2007.

[2] B. Shneiderman, et.al. Designing the user interface: strategies for effective human-computer interaction, Hoboken: Pearson, 2016.

[3] S. Serdiuk. Erhonomichni pytannia proektuvannia liudyno-mashynnykh system. Zaporizhzhia: ZNTU, 2014.

[4] B. Hollifield, et.al. The high performance HMI handbook, Kalamazoo: Digital Books, 2008.

[5] A. Anokhyn. "Problemy orhanyzatsyy cheloveko-mashynnoho ynterfeisa ASU TP AES", Doklady BHUYR, no. 2, vol. 88, 2015, pp. 104-108.

[6] A. Stopakevych, et.al. "Development of computer-integrated systems for the automation of technological process of associated gas processing", Eastern-European Journal of Enterprise Technologies, no. 3, pp. 4-12, 2017.

[7] A.F. Arabadzhy, et.al. "Syntez dvukhurovnevoi systemy upravlenyia rektyfykatsyonnoi kolonnoi v tekhnolohycheskom protsesse pererabotky poputnykh neftianykh hazov". Avtomatyzatsyia tekhnolohycheskykh y byznes-protsessov, vol. 9, no.2, pp. 11-17, 2017.

[8] M. Endsley. Designing for situation awareness: an approach for user-centered design. Boca Raton, FL: CRC Press, 2011.

УДК 681.518.3

\title{
СПЕКТРАЛЬНЫЕ ПЛОТНОСТИ РЕГУЛИРУЕМЫХ ПЕРЕМЕННЫХ ТИПОВЫХ САР И ИХ АППРОКСИМАЦИЯ МАЛОПАРАМЕТРОВЫМИ МОДЕЛЯМИ
}

\author{
В.А. Хобин ${ }^{1}$, С.И. Лагерная ${ }^{2}$ \\ 1,2Одесская национальная академия пищевых технологий, Украина \\ ORCID: ${ }^{1} 0000-0003-0238-8371 ;{ }^{2} 0000-0001-8138-3653$ \\ E-mail: silaodessa@gmail.com
}

Copyright $(2017$ by author and the journal "Automation technological and business - processes". This work is licensed under the Creative Commons Attribution International License (CC BY). http://creativecommons.org/licenses/by/4.0/

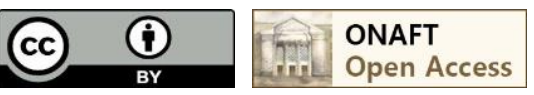

Анотация: В статье рассматриваются две группы математических моделей, которые описывают изменения регулируемых переменных замкнутых систем автоматического регулирования как случайных прочессов. Первая группа - содержательные модели, полученные на основе аналитических преобразований, которые отражают в модели спектральной плотности процесса все параметры моделей объекта, регулятора, возмущчений. Вторая группа - хорошо отражающие характер спектральной плотности, формальные модели, количество параметров которых минимально. Это позволяет вести их идентификаџию в реальном времени, в ходе штатного функционирования системы автоматического регулирования. Сравнение моделей, включая компьютерные эксперименты на основе имитационного моделирования, показывают потенциальную перспективность их применения для идентификации параметров моделей объектов регулирования в реальном времени и без размыкания контура регулирования систем автоматического регулирования.

Математическое описание системы автоматического регулирования в статье ведется, учитывая особенности объектов управления технологического типа - наличие запаздывания в каналах управления, подверженность неконтролируемым возмущениям (интенсивным координатным и параметрическим, низкочастотным).

Подробно рассмотрена прочедура получения математического выражения содержательных моделей 
http://www.atbp.onaft.edu.ua/

спектральной плотности регулируемой переменной для тестовых характерных вариантов сочетаний различных свойств системы и возмущений.

Приведены иллюстрации особенностей спектральной плотности регулируемой переменной систем автоматического регулирования для различных типовых вариантов систем и возможности замены содержательных моделей формальными.

Abstract: Two groups of the mathematical models, which describe changes in the regulated variables of closed automatic control systems as stochastic processes, are considered in the article. The first group is the informative models, obtained on the basis of analytical transformations that represent in the model of the process spectral density all the parameters of the object, regulator and perturbation effects models. The second group is well reflecting the nature of the spectral density formal models with a minimum number of parameters. This makes it possible to identify them in the real time, during the regular operation of the automatic control system. Comparison of models, including computer experiments based on simulation show the potential promise of their application for identifying the parameters of control object models in real time and without opening the control loop of automatic control systems.

In the article the mathematical description of the automatic control system is considered taking into account the features of the technological type control objects, such as the presence of delay in the control channels, susceptibility to uncontrolled disturbances (intensive coordinate disturbances and parametric, low-frequency disturbances).

A detailed considered the procedure for obtaining a mathematical expression for the informative models of the controlled variable spectral density for test typical variants of various system properties and perturbations combinations.

The illustrations of the controlled variable of automatic control systems spectral density features are presented for various types of systems. There are also presented the possibility of replacing the informative controlled variable spectral density models with formal ones.

Ключевые слова: спектральная плотность регулируемой переменной, система автоматического регулирования, модель спектральной плотности, параметры модели объекта, формальная модель, содержательная модель, объекты технологического типа, идентификация.

Keywords: controlled variable spectral density, automatic control systems, spectral density model, parameters of the object model, formal model, informative model, technological type control objects, identification.

\section{1. Описание проблемы}

Статистические методы оценивания характеристик случайных процессов САУ и их применение в задачах идентификации и адаптации разрабатываются достаточно давно [1]. Их основное (потенциальное) преимущество возможность решения указанных задач непосредственно в ходе штатного функционирования САУ. Альтернатива им решение этих задач в специально организованных режимах работы, например, размыкание САР и тестирование объекта подачей на его вход сигналов специальной формы, перевод САР в режим автоколебаний, подача на вход САР специальных тестирующих воздействий и т. д. [2]. Очевидно, что при таком подходе решение задач идентификации и адаптации входит в противоречия с основной задачей САР - стабилизацией регулируемых переменных на их заданных значениях с высокой точностью. Проявление этого противоречия обуславливает снижение этой точности, возникновение недопустимых отклонений и, как следствие, аварийных ситуаций, а также других неблагоприятных последствий.

Основной причиной редкого применения статистических методов является, на наш взгляд, математическая сложность задачи, особенно для замкнутых САР, когда их передаточные функции достаточно сложны, а передаточные функции, описывающие свойства объекта входят как в числитель, так и в знаменатель. Ситуация заметно осложняется, если САР реализуется в системах автоматизации объектами технологического типа, т.е. объектами с запаздыванием в каналах управления, подверженных интенсивным, как правило, неконтролируемым возмущениям (координатным, не сводимым к белому шуму, и параметрическим низкочастотным, которые не прогнозируются).

2. Особенности объектов управления технологического типа

При выборе моделей объектов регулирования (OP) учитывается, что принятая модель отражает действительные свойства реального объекта приближённо. В нашем случае учитывается, что исследования проводятся для объектов технологического типа, характерными особенностями которых являются: физическая распределенность каналов управления, проявляющаяся в значительных запаздываниях реакции управляемых переменных на управляющие воздействия; большое количество факторов, существенно влияющих на работу объекта, но практически не доступных для измерения, которые проявляются как неконтролируемые возмущения и изменяют значения управляемых переменных, свойства каналов управления, оптимальные режимы работы технологических агрегатов [3].

Перечисленные особенности объектов приводят к тому, что адекватное математическое описание таких объектов является весьма сложной и длительной процедурой. Поэтому в качестве альтернативы признается и отражается в моделях «неопределенность», т.е. неполнота математического описания [4].

Практика показывает, что для исследований целесообразно неопределенности моделей разделить на три независимые составляющие: структурную, параметрическую и координатную. Структурная неопределенность состоит в принципиальной невозможности задания размерности моделей каналов ОР, соответствующим свойствам объекта, параметрическая - невозможность точного задания коэффициентов дифференциальных уравнений, координатная невозможность получения точных значений переменных и статистических оценок, вычисляемых на их основе. 
Параметрическую неопределенность задают в виде моделей неконтролируемых параметрических возмущений, структурную - введением в модель звеньев чистого запаздывания, координатную - в форме модели шумов измерения, аддитивных измеряемым координатам. [5]

Разработка моделей входных воздействий сталкивается с теми же проблемами, что и при синтезе моделей по каналам преобразования воздействий. Корректным выходом и в данном случае является исходное введение в модель неопределённостей, отражающих невозможность точного описания воздействия среды на ОР. Неопределённости, проявляющиеся, прежде всего, из-за большого количества и сложности измерения воздействий, отражают введением в модель эквивалентных неконтролируемых координатных возмущений, аддитивных управляющим, либо управляемым координатам [6]. Большое количество составляющих в неконтролируемых координатных возмущениях и их преобразование инерционными каналами ОР позволяет часто принять модель воздействий в виде гауссовского процесса [5].

В инженерной практике [3] стохастические модели входных воздействий обычно представляют в форме случайного процесса $($ СП) с аддитивными составляющими, например, $f=\bar{f}(t)+\tilde{f}(t), \quad$ где $\bar{f}(t) \quad-$ детерминированная составляющая, может описываться как периодический процесс, $\tilde{f}(t)$ - стохастическая центрированная составляющая. Некоторую трудность представляет разработка модели $\tilde{f}(t)$. Одним из практических путей в данном случае является априорное задание модели $\tilde{f}(t)$ в виде моделей её корреляционной функции (КФ) и спектральной плотности [5].

3. Цель исследования

Целью исследования является создание математической основы для идентификации параметров модели объекта регулирования в условиях нормального функционирования САР. Базой для этого будет рассмотрение моделей регулируемых переменных САР, как случайных процессов.

В статье будут рассмотрены два вида математических моделей спектральных плотностей регулируемых переменных. Первая - содержательная, полученная на основе известных зависимостей аналитически. Вторая формальная, получение которой базируется на эвристических методах.

4. Свойства САР, для которых будут получены содержательные модели

4.1 Структурная схема САР и описание переменных

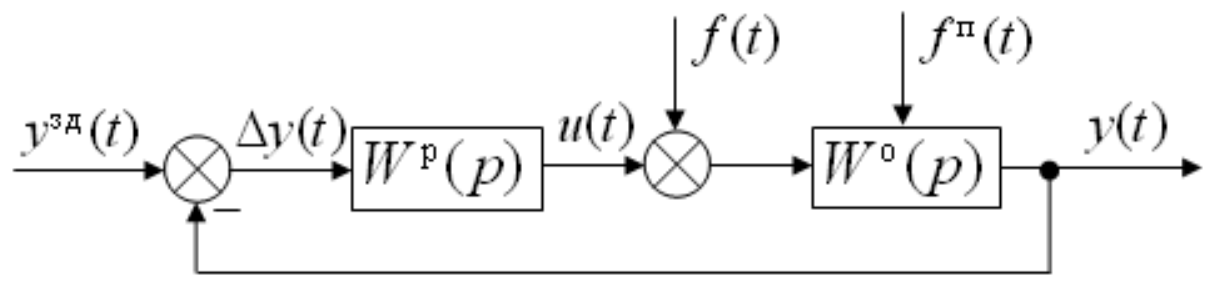

$W^{p}(p)$ - передаточная функция регулятора; $W^{o}(p)$ - передаточная функция объекта по каналу регулирования; $y(t)$ - регулируемая переменная; $y^{3 \partial}(t)$ - заданное значение регулируемой переменной;

$f(t)$-координатные возмущения; $f^{\mathrm{n}}(t)$-параметрические возмущения; $и(t)$ - управляющее воздействие; $\Delta y(t)$ - ошибка регулирования.

\section{Рис.1 - Структурная схема САР и описание переменных}

4.2 Модели ОР и особенности их параметров

С учетом изложенных особенностей объектов технологического типа, в качестве моделей каналов регулирования объектов приняты следующие [5]:

$$
\begin{gathered}
W^{\mathrm{o}}(p, t) \equiv W_{1}^{\mathrm{o}}(p, t)=\frac{k^{\mathrm{o}}(t)}{T^{\mathrm{o}}(t) \cdot p+1} e^{-\tau^{\mathrm{o}}(t) \cdot p}, \\
W^{\mathrm{o}}(p, t) \equiv W_{2}^{\mathrm{o}}(p, t)=\frac{1}{T^{\mathrm{o}}(t) \cdot p} e^{-\tau^{\mathrm{o}}(t) \cdot p},
\end{gathered}
$$

где $p \equiv \frac{d}{d t}$ - оператор дифференцирования; $W_{1}^{\mathrm{O}}(p, t)-$ передаточная функция модели ОР для каналов с самовыравниванием (статических); $W_{2}^{\mathrm{O}}(p, t)$ - передаточная функция модели ОР для каналов без самовыравнивания (астатических); $k^{\mathrm{o}}(t)$ - коэффициент передачи ОР; $T^{\mathrm{o}}(t)$ - постоянная времени ОР; $\tau^{\circ}(t)-$ время чистого запаздывания OP. 
http://www.atbp.onaft.edu.ua/

При исследовании САР с такими объектами применим метод замороженных коэффициентов [9]: за время переходного процесса параметры объекта $k^{\mathrm{o}}(t), T^{\circ}(t), \tau^{\mathrm{o}}(t)$ остаются неизменными («замороженными»). Возможность такого допущения обусловлена тем, что, как правило, параметрические возмущения являются существенно более низкочастотными, чем координатные.

4.3 Модель регулятора

В качестве регулятора принята модель ПИД-регулятора, как наиболее часто применяемая на практике [8]. ПИДрегулятор является общим случаем из типовых регуляторов. Его частным случаем можно рассматривать ПИ-, ПД-, Прегуляторы, которые могут быть получены из него, выбором соответствующих значений этих параметров.

$$
W^{\mathrm{p}}(p)=k_{p}\left(1+\frac{1}{T_{i z} p}+\frac{T_{p r} p}{0.1 T_{p r} p+1}\right)=K_{p} \frac{1,1 T_{i z} T_{p r} p^{2}+\left(0,1 T_{p r}+T_{i z}\right) p+1}{0,1 T_{i z} T_{p r} p^{2}+T_{i z} p},
$$

где $K_{p}$ - коэффициент передачи регулятора; $T_{i z}-$ время изодрома регулятора; $T_{p r}-$ время предварения регулятора.

4.4 Модели возмущений

Как показывает практика $[5,8]$, свойства реальных случайных составляющих возмущений $\tilde{f}(t)$ хорошо описываются взаимосвязанными парами корреляционных функций $(К Ф) R\left(\tau_{k}\right)$, и спектральных плотностей (СП) $S(\omega)$ :

$$
\begin{array}{cc}
R_{1}\left(\tau_{k}\right)=\sigma^{2} e^{-\alpha\left|\tau_{k}\right|}\left(1+\alpha\left|\tau_{k}\right|\right), & S_{1}(\omega)=\frac{\sigma^{2} \cdot 4 \alpha^{3}}{\left(\omega^{2}+\alpha^{2}\right)^{2}} ; \\
R_{2}\left(\tau_{k}\right)=\sigma^{2} e^{-\alpha\left|\tau_{k}\right|}\left(\cos \left(\beta\left|\tau_{k}\right|\right)+\frac{\alpha}{\beta} \sin \left(\beta\left|\tau_{k}\right|\right)\right), & S_{2}(\omega)=\frac{\sigma^{2} \cdot 4 \alpha\left(\alpha^{2}+\beta^{2}\right)}{\left(\omega^{2}-\beta^{2}-\alpha^{2}\right)^{2}+4 \alpha^{2} \omega^{2}},
\end{array}
$$

где $\tau_{\mathrm{k}}$ - время корреляции, $\omega$ - частота, $\sigma^{2}$ - дисперсия; $\alpha, \beta$ - параметры время спада и степень затухания КФ.

Важной отличительной особенностью моделей (4), (5) является то, что они соответствуют дифференцируемым случайным процессам.

4.5 Варианты САР, для которых разрабатываются содержательные модели

Таблица 1. Варианты моделей объекта регулирования, каналов регулирования, моделей возмущений, которые варьируются в моделях САР

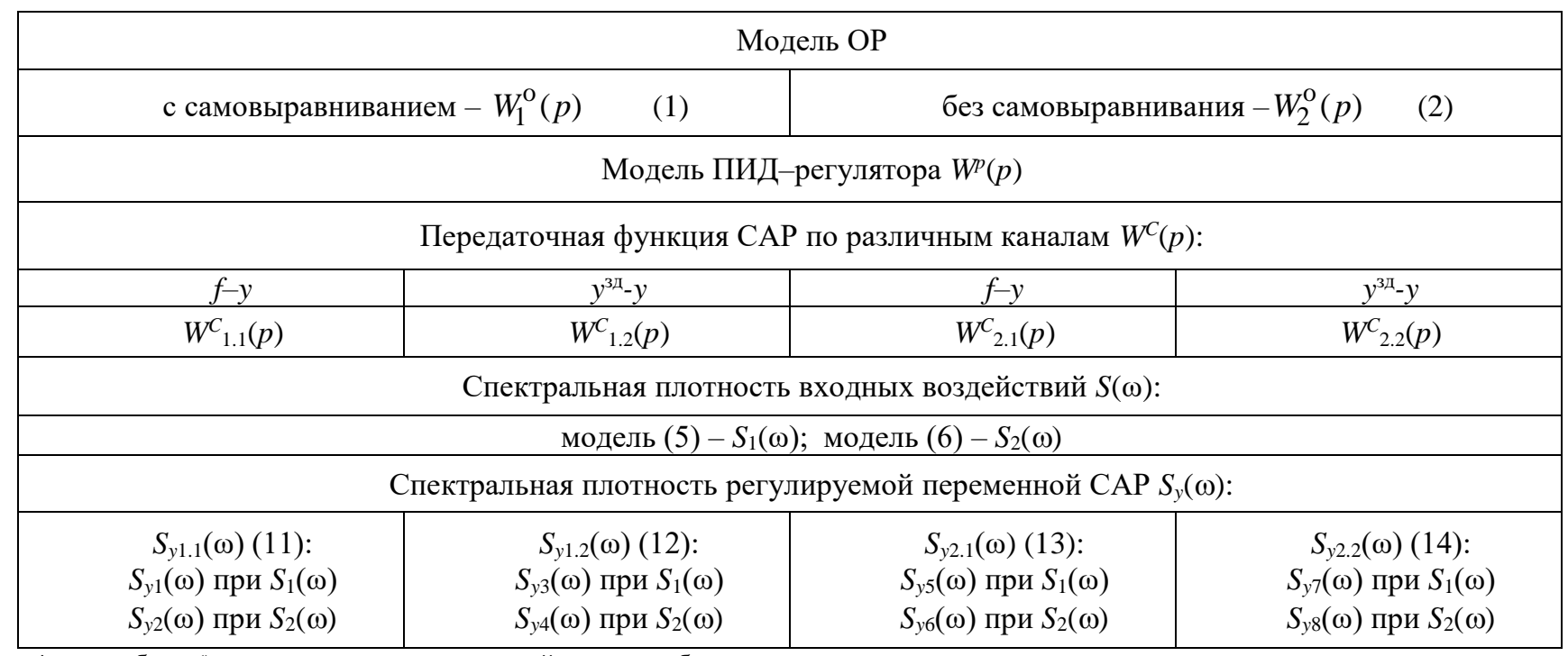

* - в скобках () указаны номера выражений, которые будут получены ниже.

5. Разработка содержательных математических моделей регулируемых переменных САР, как случайных процессов

Разработка содержательной математической модели базируется на известном соотношении, связывающем свойства замкнутой САР, координатных возмущений и регулируемой переменной как случайного процесса в частотной области [8]:

$$
S_{y}(\omega)=\left|W^{\mathrm{C}}(j \omega)\right|^{2} \cdot S(\omega),
$$

где $j \omega$ - оператор Фурье, $W^{C}(j \omega)$ - частотная передаточная функция САР, по исследуемому каналу. 
Формально процедура получения выражения $W^{C}(j \omega)$ предполагает запись передаточной функции системы в

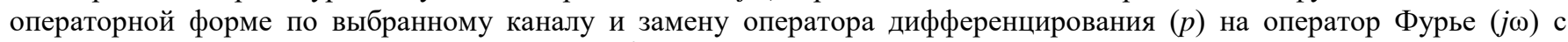
приведением подобных членов, учитывая, что $j^{2}=-1$.

Тогда

$$
\left|W^{C}(j \omega)\right|^{2}=\left|\frac{R(j \omega)}{Q(j \omega)}\right|^{2}=\frac{(\operatorname{Re} R(j \omega))^{2}+(\operatorname{Im} R(j \omega))^{2}}{(\operatorname{Re} Q(j \omega))^{2}+(\operatorname{Im} Q(j \omega))^{2}},
$$

где $R(j \omega)$ - числитель $W^{C}(j \omega), Q(j \omega)$ - знаменатель $W^{C}(j \omega)$.

Окончательно, выражение (7) принимает вид:

$$
S_{y}(\omega)=\frac{\left(\operatorname{Re} R_{y}(j \omega)\right)^{2}+\left(\operatorname{Im} R_{y}(j \omega)\right)^{2}}{\left(\operatorname{Re} Q_{y}(j \omega)\right)^{2}+\left(\operatorname{Im} Q_{y}(j \omega)\right)^{2}} \cdot S(\omega) .
$$

Иллюстрацию всей процедуры рассмотрим на одном примере получения содержательной модели спектральной плотности регулируемой переменной САР. Сформулируем особенности этого примера в соответствии с таблицей 1 из п.4.5 и принятыми там обозначениями $\quad: W_{1}^{\mathrm{O}}(p), W^{p}(p)$, канал $f-y, S(\omega)$. Тогда:

$$
\begin{gathered}
W_{1.1}^{\mathrm{c}}(p)=\frac{\frac{k^{\mathrm{o}}}{T^{\mathrm{o}} p+1} e^{-\tau^{\mathrm{o}} p}}{1+\frac{k^{\mathrm{o}}}{T^{\mathrm{o}} p+1} e^{-\tau^{\mathrm{o}} p} \cdot K_{p} \frac{1,1 T_{i z} T_{p r} p^{2}+\left(0,1 T_{p r}+T_{i z}\right) p+1}{0,1 T_{i z} T_{p r} p^{2}+T_{i z} p}}= \\
=\frac{k^{\mathrm{o}} e^{-\tau^{\mathrm{o}} p}\left(0,1 T_{i z} T_{p r} p^{2}+T_{i z} p\right)}{\left(T^{\mathrm{o}} p+1\right)\left(0,1 T_{i z} T_{p r} p^{2}+T_{i z} p\right)+k^{\mathrm{o}} K_{p} e^{-\tau^{\mathrm{o}} p}\left(1,1 T_{i z} T_{p r} p^{2}+\left(0,1 T_{p r}+T_{i z}\right) p+1\right)} . \\
W_{1.1}^{c}(j \omega)=\frac{R_{1.1}(j \omega)}{Q_{1.1}(j \omega)},
\end{gathered}
$$

где $e^{-j \omega \tau_{o}}=\cos \omega \tau_{o}-j \sin \omega \tau_{o}$

$$
\begin{aligned}
& R_{1.1}(j \omega)=k^{\mathrm{o}} \cdot\left(\cos \left(\omega \tau^{\mathrm{o}}\right)-j \sin \left(\omega \tau^{\mathrm{o}}\right)\right) \cdot\left(-0,1 T_{i z} T_{p r} \omega^{2}+T_{i z} \cdot(j \omega)\right) ; \\
& Q_{1.1}(j \omega)=\left(T^{\mathrm{o}} \cdot(j \omega)+1\right) \cdot\left(-0,1 T_{i z} T_{p r} \omega^{2}+T_{i z} \cdot(j \omega)\right)+ \\
& +k^{\mathrm{o}} K_{p} \cdot\left(\cos \left(\omega \tau^{\mathrm{o}}\right)-j \sin \left(\omega \tau^{\mathrm{o}}\right)\right) \cdot\left(-1,1 T_{i z} T_{p r} \omega^{2}+\left(0,1 T_{p r}+T_{i z}\right) \cdot(j \omega)+1\right) . \\
& S_{y 1.1}(\omega)=\frac{\left(\operatorname{Re} R_{y 1.1}(j \omega)\right)^{2}+\left(\operatorname{Im} R_{y 1.1}(j \omega)\right)^{2}}{\left(\operatorname{Re} Q_{y 1.1}(j \omega)\right)^{2}+\left(\operatorname{Im} Q_{y 1.1}(j \omega)\right)^{2}} \cdot S(\omega),
\end{aligned}
$$

где $\operatorname{Re} R_{y 1.1}(j \omega)=-0,1 k^{\circ} T_{i z} T_{p r} \cos (\omega \tau) \cdot \omega^{2}+k^{\circ} T_{i z} \sin (\omega \tau) \cdot \omega$;

$\operatorname{Im} R_{y 1.1}(j \omega)=0,1 k^{\mathrm{o}} T_{i z} T_{p r} \sin (\omega \tau) \cdot \omega^{2}+k^{\mathrm{o}} T_{i z} \cos (\omega \tau) \cdot \omega$

$\operatorname{Re} Q_{y 1.1}(j \omega)=\left(-1,1 k^{\mathrm{o}} K_{p} T_{i z} T_{p r} \cos (\omega \tau)-0,1 T_{i z} T_{p r}-T^{\mathrm{o}} T_{i z}\right) \cdot \omega^{2}+$ $+k^{\mathrm{o}} K_{p}\left(0,1 T_{i z}+T_{p r}\right) \sin (\omega \tau) \cdot \omega+k^{\mathrm{o}} K_{p} \cos (\omega \tau)$;

$\operatorname{Im} Q_{y 1.1}(j \omega)=-0,1 T^{\mathrm{o}} T_{i z} T_{p r} \cdot \omega^{3}+1,1 k^{\mathrm{o}} K_{p} T_{i z} T_{p r} \sin (\omega \tau) \cdot \omega^{2}+$ $+\left(T_{i z}+k^{\mathrm{o}} K_{p}\left(0,1 T_{p r}+T_{i z}\right) \cos (\omega \tau)\right) \cdot \omega-k^{\mathrm{o}} K_{p} \sin (\omega \tau)$. 
Реализация указанной процедуры для других вариантов САР из табл.1 дает следующие результаты: (12) (14). Для $W_{1}^{\mathrm{o}}(p), W^{p}(p)$, канал $y^{\text {зд }}-y, S(\omega)$ :

$$
S_{y 1.2}(\omega)=\frac{\left(\operatorname{Re} R_{y 1.2}(j \omega)\right)^{2}+\left(\operatorname{Im} R_{y 1.2}(j \omega)\right)^{2}}{\left(\operatorname{Re} Q_{y 1.2}(j \omega)\right)^{2}+\left(\operatorname{Im} Q_{y 1.2}(j \omega)\right)^{2}} \cdot S(\omega),
$$

где $\operatorname{Re} R_{y 1.2}(j \omega)=-1,1 k^{\mathrm{o}} K_{p} T_{i z} T_{p r} \cos (\omega \tau) \cdot \omega^{2}+\left(0,1 T_{p r}+T_{i z}\right) k^{\mathrm{o}} K_{p} \sin (\omega \tau) \cdot \omega+k^{\mathrm{o}} K_{p} \cos (\omega \tau)$;

$\operatorname{Im} R_{y 1.2}(j \omega)=1,1 k^{\mathrm{o}} K_{p} T_{i z} T_{p r} \sin (\omega \tau) \cdot \omega^{2}+\left(0,1 T_{p r}+T_{i z}\right) k^{\mathrm{o}} K_{p} \cos (\omega \tau) \cdot \omega-k^{\mathrm{o}} K_{p} \sin (\omega \tau) ;$

$\operatorname{Re} Q_{y 1.2}(j \omega)=\left(-T^{\mathrm{o}} T_{i z}-0.1 T_{i z} T_{p r}-1,1 k^{\mathrm{o}} K_{p} T_{i z} T_{p r} \cos (\omega \tau)\right) \cdot \omega^{2}+\left(0,1 T_{p r}+T_{i z}\right) k^{\mathrm{o}} K_{p} \sin (\omega \tau) \cdot \omega+$ $+k^{\mathrm{o}} K_{p} \cos (\omega \tau)$

$\operatorname{Im} Q_{y 1.2}(j \omega)=-0.1 T^{\mathrm{o}} T_{i z} T_{p r} \cdot \omega^{3}+\left(T_{i z}+1,1 k^{\mathrm{o}} K{ }_{p} T_{i z} T_{p r} \sin (\omega \tau)\right) \cdot \omega^{2}+$ $+\left(0,1 T_{p r}+T_{i z}\right) k^{\mathrm{o}} K_{p} \cos (\omega \tau) \cdot \omega-k^{\mathrm{o}} K_{p} \sin (\omega \tau)$.

Для $W_{2}^{\mathrm{o}}(p), W^{p}(p)$, канал $f-y, S(\omega)$ :

$$
S_{y 2.1}(\omega)=\frac{\left(\operatorname{Re} R_{y 2.1}(j \omega)\right)^{2}+\left(\operatorname{Im} R_{y 2.1}(j \omega)\right)^{2}}{\left(\operatorname{Re} Q_{y 2.1}(j \omega)\right)^{2}+\left(\operatorname{Im} Q_{y 2.1}(j \omega)\right)^{2}} \cdot S(\omega),
$$

где $\operatorname{Re} R_{y 2.1}(j \omega)=-0,1 T_{i z} T_{p r} \cos (\omega \tau) \cdot \omega^{2}+T_{i z} \sin (\omega \tau) \cdot \omega$;

$\operatorname{Im} R_{y 2.1}(j \omega)=0,1 T_{i z} T_{p r} \sin (\omega \tau) \cdot \omega^{2}+T_{i z} \cos (\omega \tau) \cdot \omega$

$\operatorname{Re} Q_{y 2.1}(j \omega)=\left(-T^{\mathrm{o}} T_{i z}-1,1 K_{p} T_{i z} T_{p r} \cos (\omega \tau)-0,1 T_{i z} T_{p r}-T^{\mathrm{o}} T_{i z}\right) \cdot \omega^{2}+$

$+\left(K_{p} T_{i z} \sin (\omega \tau)+0,1 K_{p} T_{p r} \sin (\omega \tau)\right) \cdot \omega+K_{p} \cos (\omega \tau)$;

$\operatorname{Im} Q_{y 2.1}(j \omega)=-0,1 T^{\mathrm{o}} T_{i z} T_{p r} \omega^{3}+1,1 K_{p} T_{i z} T_{p r} \sin (\omega \tau) \omega^{2}+$ $+\left(K_{p} T_{i z} \cos (\omega \tau)+0,1 K_{p} T_{p r} \cos (\omega \tau)\right) \omega-K_{p} \sin (\omega \tau)$.

Для $W_{2}^{\mathrm{o}}(p), W^{p}(p)$, канал $y^{\text {зд- }}-y, S(\omega)$ :

$$
S_{y 2.2}(\omega)=\frac{\left(\operatorname{Re} R_{y 2.2}(j \omega)\right)^{2}+\left(\operatorname{Im} R_{y 2.2}(j \omega)\right)^{2}}{\left(\operatorname{Re} Q_{y 2.2}(j \omega)\right)^{2}+\left(\operatorname{Im} Q_{y 2.2}(j \omega)\right)^{2}} \cdot S(\omega),
$$

где $\operatorname{Re} R_{y 2.2}(j \omega)=-1,1 T_{i z} T_{p r} K_{p} \cos (\omega \tau) \cdot \omega^{2}+\left(0.1 T_{p r}+T_{i z}\right) K_{p} \sin (\omega \tau) \cdot \omega+K_{p} \cos (\omega \tau)$;

$\operatorname{Im} R_{y 2.2}(j \omega)=1,1 T_{i z} T_{p r} K_{p} \sin (\omega \tau) \cdot \omega^{2}+\left(0,1 T_{p r}+T_{i z}\right) K_{p} \cos (\omega \tau) \cdot \omega-K_{p} \sin (\omega \tau) ;$

$\operatorname{Re} Q_{y 2.2}(j \omega)=\left(-T_{i z} T^{\mathrm{o}}-1,1 T_{i z} T_{p r} K_{p} \cos (\omega \tau)\right) \cdot \omega^{2}+\left(0.1 T_{p r}+T_{i z}\right) K_{p} \sin (\omega \tau) \cdot \omega+K_{p} \cos (\omega \tau) ;$

$\operatorname{Im} Q_{y 2.2}(j \omega)=-0.1 T_{i z} T_{p r} T^{\mathrm{o}} \omega^{3}+1,1 T_{i z} T_{p r} K_{p} \sin (\omega \tau) \cdot \omega^{2}+\left(0,1 T_{p r}+T_{i z}\right) K_{p} \cos (\omega \tau) \cdot \omega-K_{p} \sin (\omega \tau)$.

Стоит отметить, что спектральная плотность регулируемой переменной САР при одновременно действующих входных сигналах $f(t)$ и $y^{3 \partial}(t)$ равна алгебраической сумме спектральных плотностей:

$$
S_{y}(\omega)=\left|W_{f-y}^{c}(j \omega)\right|^{2} \cdot S_{f}(\omega)+\left|W_{y^{c \breve{a}}-y}^{c}(j \omega)\right|^{2} \cdot S_{y^{c} \ddot{a}}(\omega),
$$

где $W_{f-y}^{c}(j \omega), W_{y^{c ̧ a ̈}-y}^{c}(j \omega)$ - частотные передаточные функции САР по каналу возмущений и задания соответственно; $S_{f}(\omega)$ - спектральная плотность входного воздействия по каналу возмущений, $S_{y c ̧ \ddot{a}}(\omega)-$ спектральная плотность входного воздействия по каналу задания. 
http://www.atbp.onaft.edu.ua/

6. Иллюстрация особенностей $S_{y}(\omega)$ для различных характерных тестовых вариантов

Для иллюстрации особенностей спектральной плотности регулируемой переменной САР рассматривались тестовые характерные варианты сочетаний различных свойств САР и возмущений, см. рис. 2, 3.

Необходимо отметить, что частотный состав возмущающих воздействий выбирался с учетом предельной частоты исследуемых САР [7], в которых они способны эффективно подавлять внешние возмущения.

Настроечные параметры регуляторов тестовых САР были выбраны так, чтоб рассмотреть альтернативные варианты: колебательный переходный процесс и апериодический переходный процесс.

7. Обоснование вида формальной малопараметровой модели

Полученные модели (12) - (14) не удобны для практического применения. Они содержат большое количество параметров и достаточно громоздкие. Анализируя полученные графики моделей спектральных плотностей регулируемых переменных замкнутых САР видно, что они имеют общие характерные особенности: для канала $f-y$ (рис.2) значение спектральной плотности на низких частотах стремится к нулевому значению. Эта особенность позволяет сделать вывод о том, что такие модели не корректно описывать моделями вида (4), (5) одно-, двухпараметровыми. Рассмотрим модель, которая отражает особенности топологии спектральных плотностей регулируемых переменных и содержит три параметра, которые подлежат идентификации:

$$
S_{y}(\omega)=\frac{\sigma_{y}{ }^{2} 4 \alpha \omega^{2}\left(\left(\alpha^{2}+\beta^{2}\right) \gamma^{2}+2 \alpha \gamma+1\right)}{\left(1+\gamma^{2} \omega^{2}\right)\left(\left(\omega^{2}-\beta^{2}-\alpha^{2}\right)^{2}+4 \alpha^{2} \omega^{2}\right)}
$$

где $\alpha, \beta, \gamma-$ параметры.

Предложенная модель хорошо описывает спектральные плотности регулируемых переменных САР. Это доказывает аппроксимация, проведенная численными методами. В качества критерия близости моделей принят интегральный квадратичный критерий. Значения критерия после оптимизации в каждом случае меньше 0,001 .

Иллюстрации, демонстрирующие то, что для всех тестовых вариантов подбором параметров удается обеспечить высокую степень приближения приведены на рис. 4.

По каналу $y^{3 д}-y$ (рис. 3) спектральная плотность регулируемой переменной сходна со спектральной плотностью $y^{3 \partial}(t)$. В этом случае, в качестве модели $S_{y}(\omega)$ целесообразно выбрать двухпараметровую модель (5).

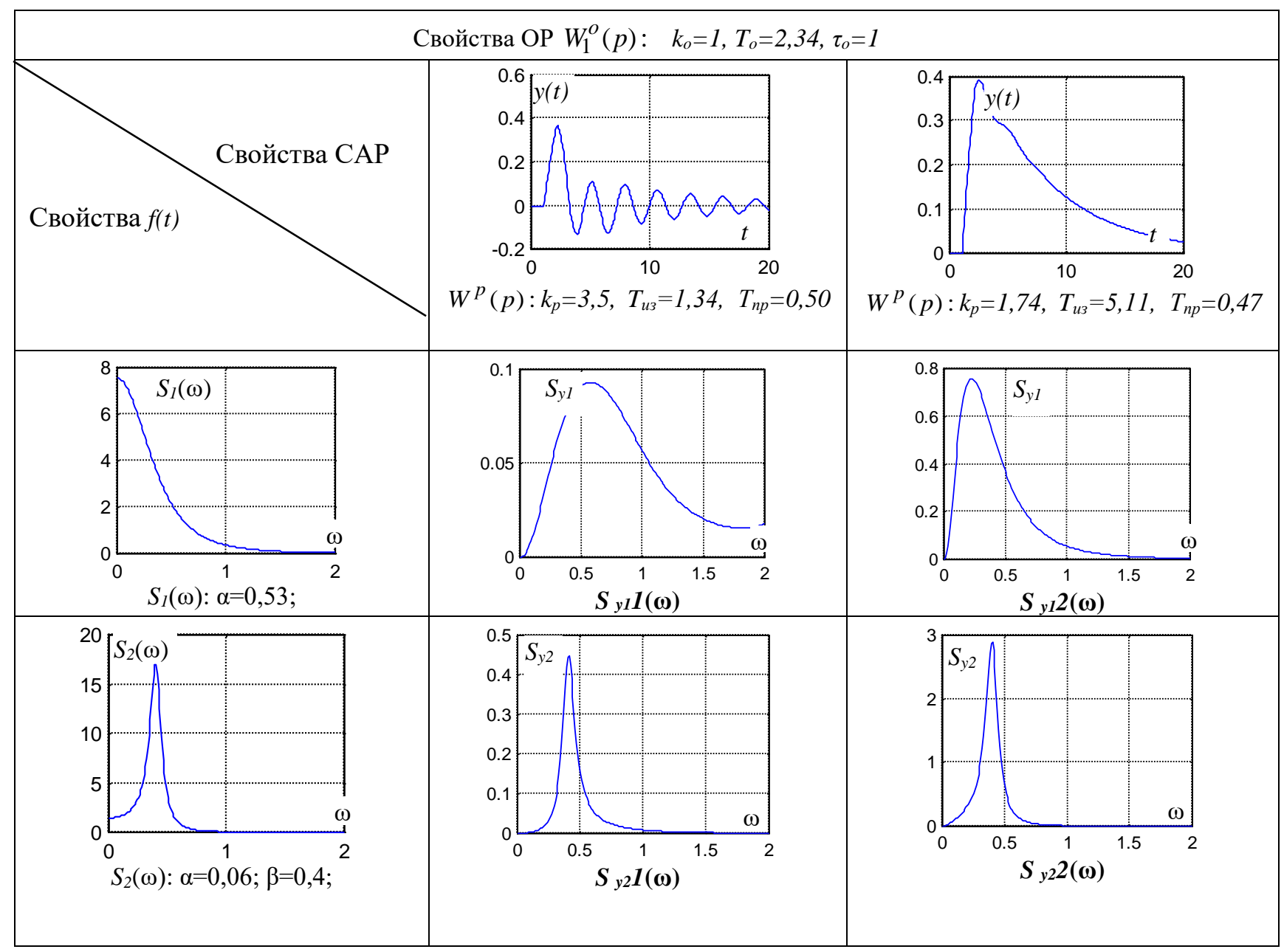




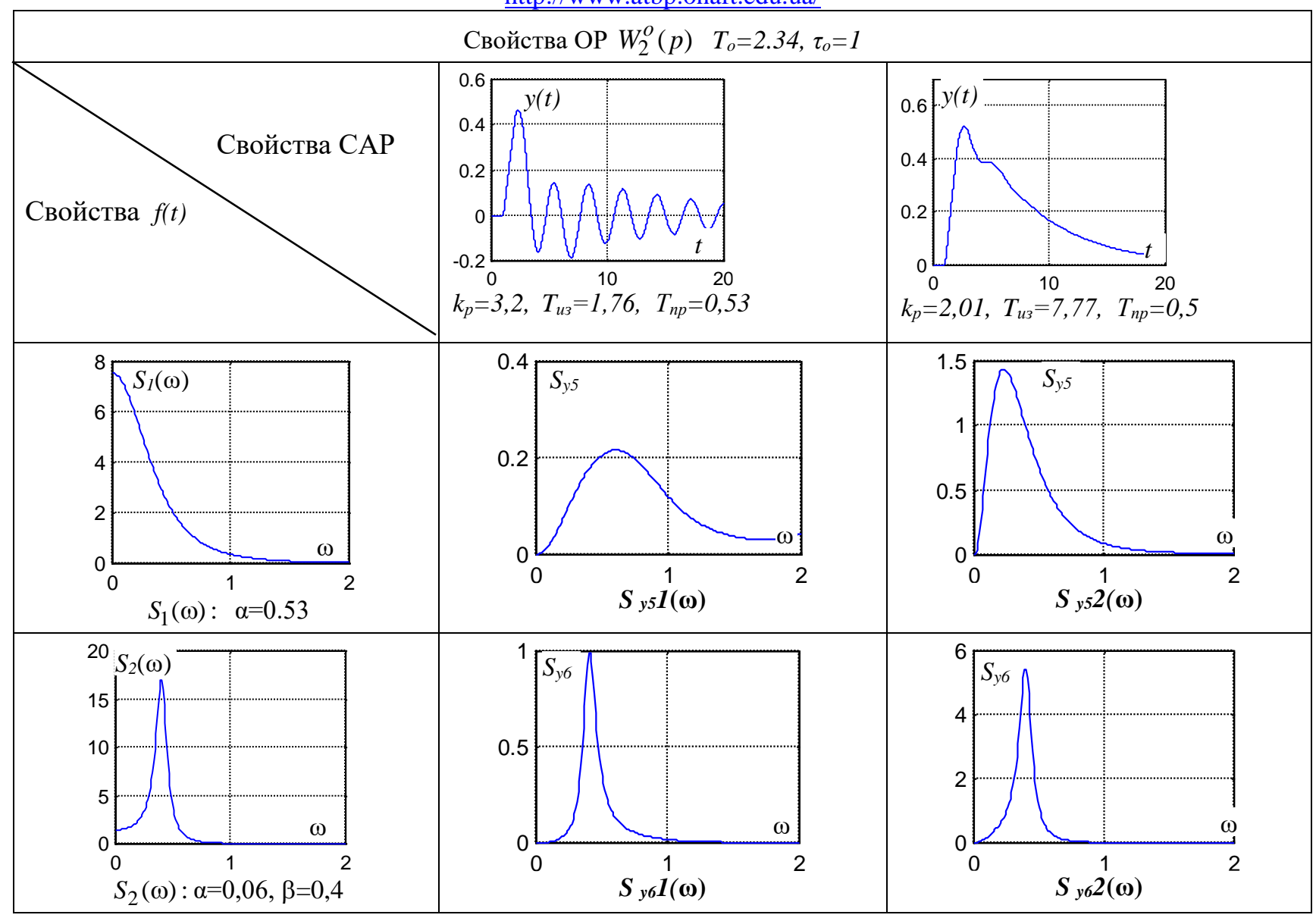

Рис. 2 - Иллюстрация свойств САР, возмущений, спектральной плотности регулируемой переменной

Свойства $y^{30}(t)$




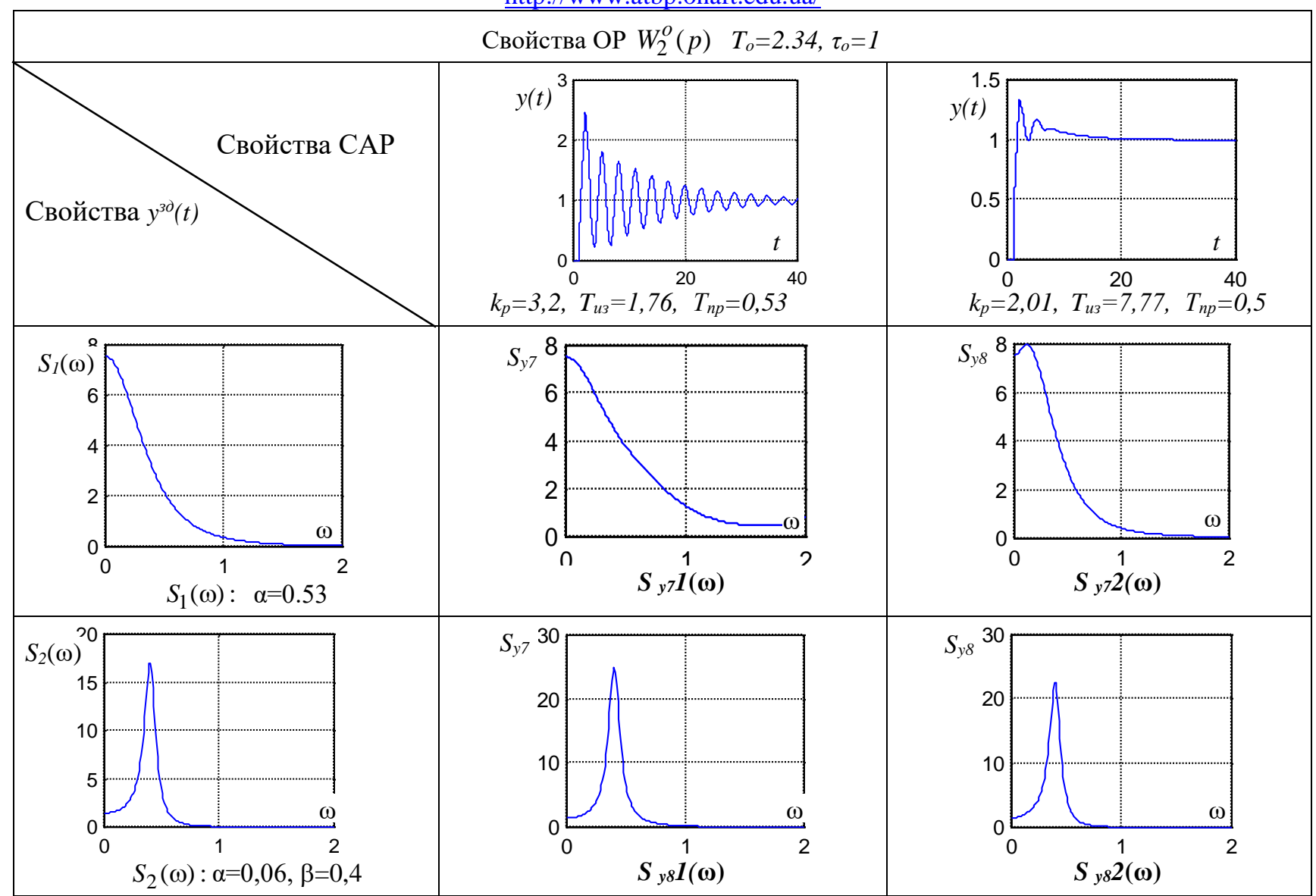

Рис. 3 - Иллюстрация свойств САР, заданного значения регулируемой переменной, спектральной плотности регулируемой переменной
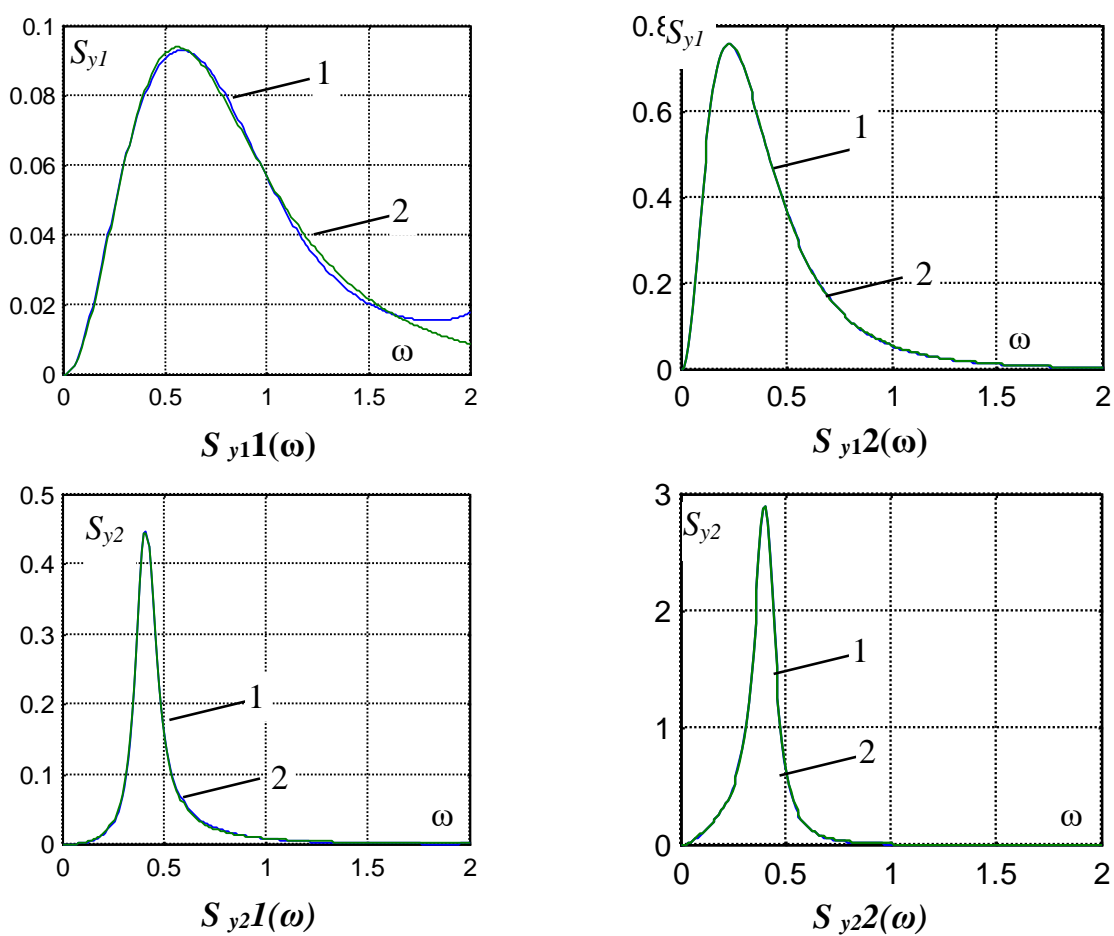

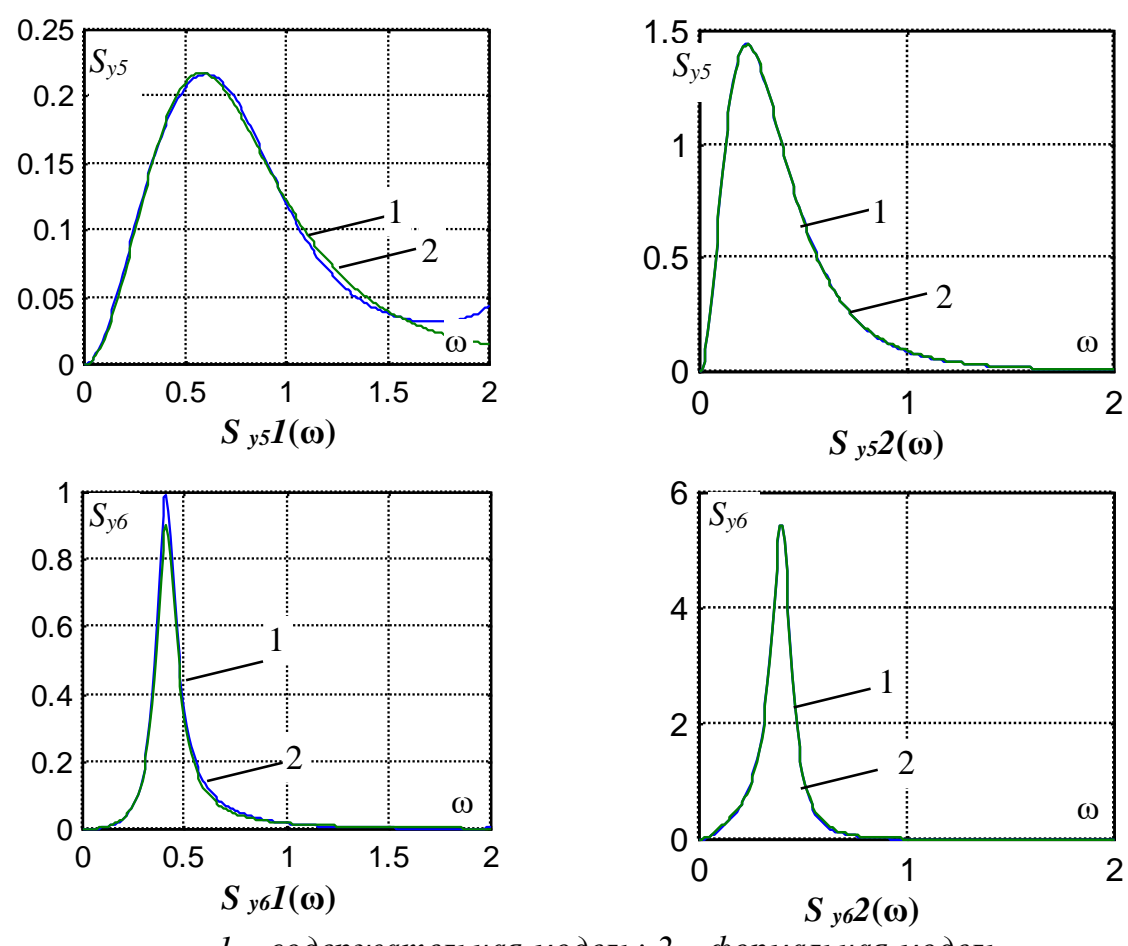

1 - содержательная модель; 2 - формальная модель

Рис. 4 - Иллюстрация особенностей $S_{y}(\omega)$ для тестовых вариантов и возможности замены содержательных моделей (11), (13) формальной (15)
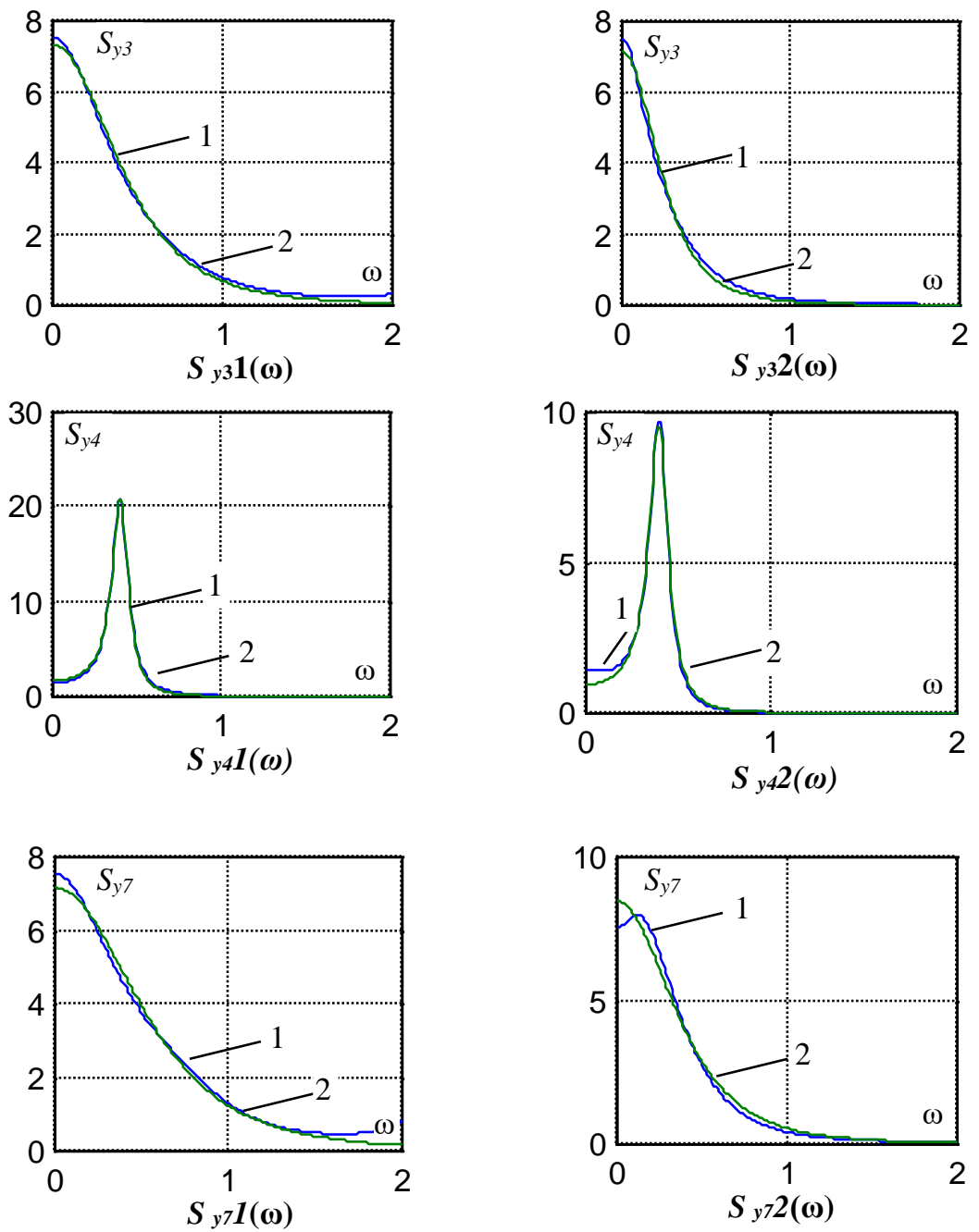
http://www.atbp.onaft.edu.ua/

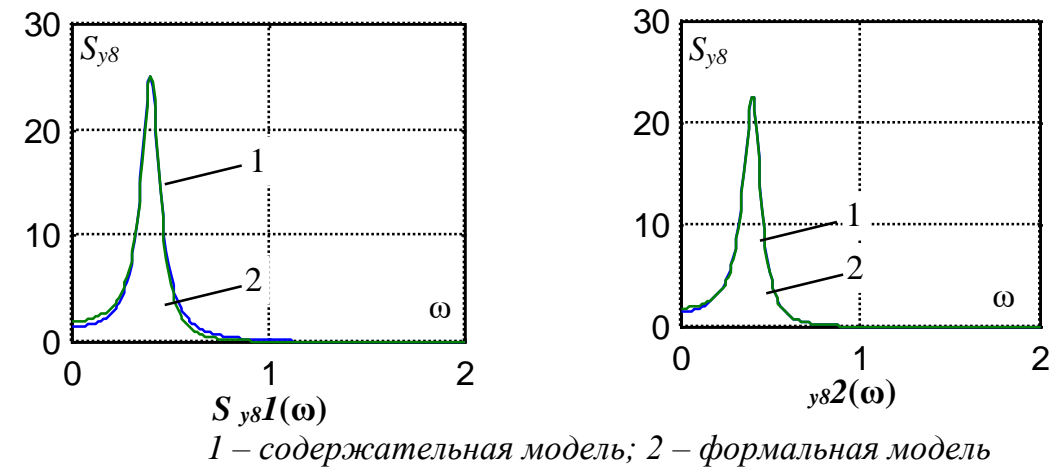

Рис. 5 - Иллюстрация особенностей $S_{y}(\omega)$ для тестовых вариантов и возможности замены содержательных моделей (12), (14) формальной (4)

\section{8. Выводы}

Содержательные модели сложны и их «применение» для решения задач оценивания и идентификации в реальном времени требует выделения больших вычислительных ресурсов контроллеров и достаточно сложного программного обеспечения.

Проведенные исследования показывают, что предложенные малопараметровые модели могут хорошо описывать содержательные. Для их идентификации могут быть использованы разработанные методы, например метод среднеквадратических частот. Они ориентированы на работу в реальном времени и требуют минимум ресурсов.

Переход к обратной задаче, т.е. подстройке содержательных моделей, включающих в себя неизвестные параметры объекта управления $\left(k^{\mathrm{o}}(t) ; T^{\mathrm{o}}(t) ; \tau^{\mathrm{o}}(t)\right)$ может позволить решать задачу параметрической идентификации. Оценка эффективности ее практического применения - задача последующих исследований.

\section{Литература}

[1] Пугачев В.С., Основы статистической теории автоматических систем / В.С. Пугачев, И.Е. Казаков, Л.Г. Евланов. - М., «Машиностроение», 1974. - 400 с.

[2] Бессонов, А.А. Методы и средства идентификации динамических объектов / А.А. Бессонов, Ю.В. Загашвили, А.С. Маркелов. - Л.: Энергоатомиздат. Ленингр. отд-ние, 1989.- 280 с.

[3] Хобин, В.А. Системы гарантирующего управления технологическими агрегатами: основы теории, практика применения. / В.А. Хобин. - О., Одесская национальная академия пищевых технологий, «ТЭС», 2008 - 306 с.

[4] Штейнберг, Ш.Е. Идентификация в системах управления. - М.: Энергоатомиздат, 1987. - 80 с.

[5] Жигайло, А.М. Статистическая идентификация моделей динамики и ее применение в задачах адаптивного управления: Дисс. ... канд. техн. наук: 05.13.07. - Одесса, 2001. - 174 с.

[6] Солодовников, В.В., Дмитриев, А.Н., Егупов, Н.Д. Спектральные методы расчёта и проектирования систем управления. / В.В. Солодовников, А.Н. Дмитриев, Н.Д. Егупов - М.: Машиностроение, 1986.- 440 с.

[7] Левинский, М.В. Тестовые САР для исследования алгоритмов их самонастройки / М.В. Левинский - Наукові праці ОНАХТ - 2015. - Випуск 48. - с. 142 - 146.

[8] Бесекерский, В.А. Теория систем автоматического управления / В.А. Бесекерский, Е.П. Попов // Изд. 4-е, переработанное и доп.- СПб., Профессия, 2007. - 725 с.

[9] Попов, Д.Н. Динамика и регулирование гидро- и пневмосистем / Д.Н. Попов // Изд. 2-е, переработанное и доп. - М. Машиностроение, 1987. - 464 с.

\section{References}

[1] Pugachev V.S., Osnovy statisticheskoy teoriy avtomaticheskih system. V.S. Pugachev, I.E. Cazakov, L.G. Evlanov, Moskow: Machinistroenie, 1974.

[2] Bessonou A.A., Metody I sredstva identificacii dinamycheskih obectov. A.Bessonou, U. Zagachvily, A. Markelou, Leningrad: Energoatomizdat. Lening. otdelenie, 1989.

[3] Khobin V.A., Systemy garantiruushego upravleniya technologicheskymy agregatamy: osnovy teoryi, practica primeneniya. V.A. Khobin, Odessa: ONAFT, TES, 2008.

[4] Shteinberg Sh. E., Identifikaciya v systemah upravleniya. Moscow: Energoatomizdat, 1987.

[5] Jigaylo A.M. "Statisticheskaya identifikaciya modeley dynamyky I eye primeneniye v zadachah adaptivnogo upravleniya" Ph.D dissertation. 05.13.07. Odessa, 2001.

[6] Solodovnikou V.V. Spectralniye metody rascheta I proectirovaniya system upravleniya. V.V. Solodovnikou, A.N. Dmytriev, N. D. Yegupou. Moscow: Mashinostroyenie, 1986.

[7] Levinsky M.V. Testoviye SAR dlya issledovaniya algoritmov ih samonastroyki. Naukovy praci ONAHT, 2015, Vipusk 48. pp. 142 - 146. 
[8] Besekersky V.A. Teoriya system avtomaticheskogo upravleniya. V.A. Besekerskyi. St.Peterburg: Professiya, 2007.

[9] Popov D.N. Dynamyka I regulirovaniye gydro I pnevmosystem. M.: Mashinostroyeniye, 1987.

\title{
СИСТЕМА АВТОМАТИЧЕСКОГО УПРАВЛЕНИЯ БЕСПИЛОТНОГО ЛЕТАТЕЛЬНОГО АППАРАТА
}

\author{
К.О. Габуев ${ }^{1}$, В.О. Гонгало ${ }^{2}$, Н.А. Кучеренко ${ }^{3}$, А.И. Шипко ${ }^{4}$ \\ 1,2,3,4Одесская национальная академия пищевых технологий \\ E-mail: ${ }^{1}$ kostyacart@gmail.com, ${ }^{2}$ skipper12@gmail.com, ${ }^{3}$ tala08@ukr.net, ${ }^{4}$ shipko.arkadiy@gmail.com
}

Copyright $(\mathcal{C} 2017$ by author and the journal "Automation technological and business - processes". This work is licensed under the Creative Commons Attribution International License (CC BY). http://creativecommons.org/licenses/by/4.0/

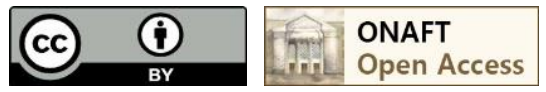

Анотация: В статье рассматривается применение беспилотных летательных аппаратов как в промышленности в иелом, так и в отдельных ее отраслях. Более подробно описывается эксплуатация беспилотных летательных аппаратов в агросекторе. Приведены проблемы, существующие на сегодняшний день в этом секторе, а также методы решения этих проблем при помощи беспилотных технологий. Объяснена важная взаимосвязь между системой автоматического управления и ее интеграцией на беспилотные летательные аппараты. Подробно описывается техническая составляющая дрона, а также взаимосвязь между каждым звеном технического средства автоматизаџии. Приведен пример программного обеспечения, через которое осуществляется управление беспилотным летательным аппаратом.

Abstract: The article deals with the use of unmanned aerial vehicles as in industry in general and as in individual branches. The operation of unmanned aerial vehicles in the agricultural sector is described in more detail. The problems existing for today in this sector, as well as the methods of solving these problems with the help of unmanned technologies are given. The important interrelation between the automatic control system and its integration into unmanned aerial vehicles is explained. Detailed description of the technical component of the drone, as well as the relationship between each link of the technical means of automation. An example of the software through which the unmanned aerial vehicle is controlled is given.

Ключевые слова: робот, дрон, робототехника, автоматизация, система автоматического управления, математическая модель.

Keywords: robot, drone, robotics, automation, automatic control system, mathematical model.

\section{Вступление}

На сегодняшний день технологии по всему миру стремительно развиваются, в том числе и автоматизация, технологический прорыв в индустриализации и в секторах, которые с ней связаны, позволяют смотреть по-новому на существующие проблемы и решать их новыми современными, а также актуальными способами. Например, применение беспилотных технологий в аграрном и промышленном секторах [1] [2] - это одна из новых и очень актуальных технологий, которая еще была недоступна 10 лет назад и только начинала развиваться в то время. Беспилотные технологии настолько универсальны, что могут решать достаточно много проблем в разных отраслях, таких как: сельское хозяйство, добывающая промышленность, геодезия, топография, нефтегазовая промышленность, телекоммуникация, оперативное страхование, энергоэффективность, транспортировка и т.д.

В Украине, как и во всем мире в целом, наиболее активным использованием беспилотных технологий - является аграрный сектор. Внедрение дронов в сельское хозяйство - решает целый ряд проблем, например, таких как: мониторинг сельхоз угодий на наличие подтоплений территории, контроль равномерности посева, прогноз урожайности, борьба с вредителями урожая, распыление удобрений, полив и т.д. Решение всех выше перечисленных 